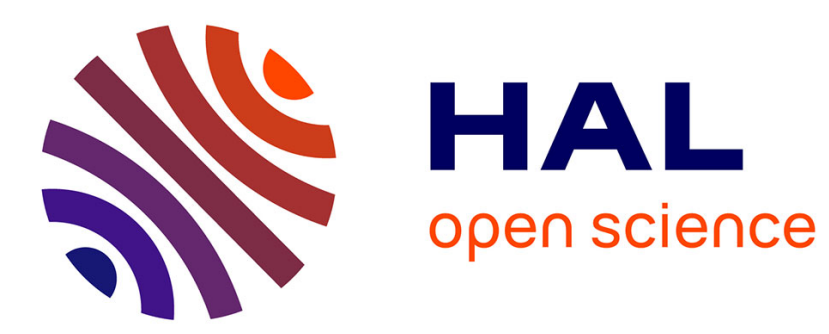

\title{
A comparative study of reduced-order modeling techniques for nonlinear MEMS beams
}

\author{
Jerome Juillard
}

\section{To cite this version:}

Jerome Juillard. A comparative study of reduced-order modeling techniques for nonlinear MEMS beams. DTIP 2014, Apr 2014, Cannes, France. pp.261-265. hal-01083889

\section{HAL Id: hal-01083889}

\section{https://hal-centralesupelec.archives-ouvertes.fr/hal-01083889}

Submitted on 18 Nov 2014

HAL is a multi-disciplinary open access archive for the deposit and dissemination of scientific research documents, whether they are published or not. The documents may come from teaching and research institutions in France or abroad, or from public or private research centers.
L'archive ouverte pluridisciplinaire HAL, est destinée au dépôt et à la diffusion de documents scientifiques de niveau recherche, publiés ou non, émanant des établissements d'enseignement et de recherche français ou étrangers, des laboratoires publics ou privés. 
A comparative study of reduced-order modeling techniques for nonlinear MEMS beams

Jérôme Juillard - E3S - SUPELEC - FRANCE

\begin{abstract}
This paper is dedicated to the comparison of three techniques of reduced-order modeling (ROM) that may be applied to MEMS beams subject to nonlinear damping and restoring forces. These methods are compared in terms of simplicity and accuracy, in the static, transient and steady-state regimes. It is shown that one of the most popular ROM methods may lead to dramatically wrong results in the case of single-mode decomposition.
\end{abstract}

\title{
Keywords
}

Reduced-order modeling; electrostatic nonlinearity.

\section{1 - Introduction}

This paper is dedicated to the comparison of three techniques of reduced-order modeling (ROM) that may be applied to MEMS beams subject to nonlinear damping and restoring forces. A difficulty which arises in this context is that the nonlinear forces acting on the beams are not polynomial. For example, consider the single-sided electrostatic actuation of a beam with Young's modulus $E$, density $\rho$, length $L$, width $b$, electrostatic gap $G$, moment of inertia $I$, and damping coefficient $\mu$, governed by:

$E I \frac{\partial^{4} w}{\partial x^{4}}+\mu \frac{\partial w}{\partial t}+\rho b h \frac{\partial^{2} w}{\partial t^{2}}=\frac{\varepsilon_{0} b}{2 G^{3}} \frac{V(t)^{2}}{(1-w)^{2}}$,

where $G w(x, t)$ is the displacement of the beam. To generate a lumped model from (1), modal projection methods must be used: to this end, it is assumed that

$w(x, t) \approx \hat{w}(x, t)=\sum_{k=1}^{N} a_{k}(t) w_{k}(x)$

in (1). If a classical Galerkin technique is used, (1) is then projected on the basis formed by the shape functions $w_{k}(x)$, typically the natural eigenmodes of the beam. The difficulty with this approach is that there exist no closed-form expressions for the projection integrals appearing on the right-hand side, even in the case when $N=1$. Among the techniques for dealing with this issue, we shall focus on the following three:

- Taylor series (TS) expansion of the nonlinear terms of (1), as used in [1-3], for example. This has the obvious advantage of transforming the non-polynomial nonlinearity into a polynomial one. However, the range of validity of the approximation is limited by the order of the truncation.

- Multiplication of (1) by $(1-w)^{2}$ before projection (MBP) on the set of shape functions, as proposed in [4-5]. As the TS approach, this increasingly popular technique (see [6-8] for example) has the advantage of turning a non-polynomial nonlinearity into a polynomial one and has full-gap range of validity, provided $N$ is sufficiently large. However, for a number of reasons, most authors using this technique limit themselves to the $N=1$ case, as in [7]. 
- Approximation of the projection integrals (API), typically by a rational function (as proposed in [9]) or by a function with the same asymptotic behavior as the projection integrals as the gap closes [10]. The latter approach is quite simple to apply in the case one eigenmode is dominant in the response of the beam. It is illustrated in [10] in the case of the electrostatic actuation of a beam switch with squeeze-film damping.

In this paper, we will focus on the use of these methods in the case of a clamped-clamped beam resonator. These methods are compared in terms of simplicity and accuracy, in the transient and steady-state regimes. It is shown that the MBP method may lead to dramatically wrong results in the case of single-mode decomposition. Some guidelines are given for solving for the steady-state responses in the three cases.

\section{2 - Lumped model of the clamped-clamped resonator}

Letting $\xi=x / L$ and $\tau=\omega_{0} t$, where

$\omega_{0}^{2}=\frac{\lambda^{4} E I}{\rho b h L^{4}}, \quad \lambda \approx 4.73,(3)$

supposing $V(\tau)=V_{b}\left(1+v_{0} \cos (\omega \tau)\right)$, where $v_{0}<<1$, and $w(\xi, \tau) \approx a(\tau) w_{C C}(\xi)$ with $w_{C C}(\xi)$ the first clamped-clamped eigenmode, (1) can be rewritten as:

$$
\left(a+\frac{\dot{a}}{Q}+\ddot{a}\right) w_{C C}=\eta \frac{1+2 v_{0} \cos (\omega \tau)}{\left(1-a w_{C C}\right)^{2}}
$$

where the dot denotes differentiation with respect to $\tau, \eta$ is an electromechanical coupling coefficient and $Q$ is the quality factor.

\section{TS method}

The right-hand side of (4) is expanded assuming $a w_{C C}<<1$. For a third order expansion, this yields:

$$
\left(a+\frac{\dot{a}}{Q}+\ddot{a}\right) w_{C C}=\eta\left(1+2 v_{0} \cos (\omega \tau)\right)\left(1+2 a w_{C C}+3\left(a w_{C C}\right)^{2}+4\left(a w_{C C}\right)^{3}\right)
$$

Projection on $w_{C C}$ results in the transient lumped model:

$\left(a+\frac{\dot{a}}{Q}+\ddot{a}\right) I_{1}=\eta\left(1+2 v_{0} \cos (\omega \tau)\right)\left(I_{0}+2 a I_{1}+3 a^{2} I_{2}+4 a^{3} I_{3}\right),(6)$

where $I_{k}=\int_{0}^{1}\left(w_{C C}\right)^{k+1} d \xi\left(I_{0} \approx 0.523, I_{1} \approx 0.397, I_{2} \approx 0.332\right.$ and $\left.I_{3} \approx 0.291\right)$.

The steady-state model is obtained by assuming that $a(\tau)=A \sin (\omega \tau+\varphi)$ and projecting (6) on $\sin (\omega \tau+\varphi)$ and $\cos (\omega \tau+\varphi)$ (i.e. by averaging over one period [11]). This yields the following equations: 


$$
\left\{\begin{array}{c}
A\left[\left(1-\omega^{2}\right)-\eta\left(2+3 \frac{I_{3}}{I_{1}} A^{2}\right)\right]=-2 \eta v_{0} \sin \varphi \frac{I_{0}}{I_{1}}\left(1+\frac{9}{4} \frac{I_{2}}{I_{0}} A^{2}\right) \\
A \frac{\omega}{Q}=2 \eta v_{0} \cos \varphi \frac{I_{0}}{I_{1}}\left(1+\frac{3}{4} \frac{I_{2}}{I_{0}} A^{2}\right)
\end{array} .\right.
$$

For a given value of $v_{0}$, one may eliminate $\sin \varphi$ and $\cos \varphi$ from (7) and express $A^{2}$ as the root of a $5^{\text {th }}$-degree polynomial, which is how the steady-state equations are usually solved. Equivalently, the following technique may be used for plotting the frequency response, with a lesser computational burden. Express $v_{0}$ as a function of $A$ and $\omega$ by eliminating $\sin \varphi$ and $\cos \varphi$ from (7):

$$
v_{0}=\frac{I_{1}}{2 \eta I_{0}} A\left(\frac{\omega^{2}}{Q^{2}\left(1+\frac{3}{4} \frac{I_{2}}{I_{0}} A^{2}\right)^{2}}+\frac{\left[\left(1-\omega^{2}\right)-\eta\left(2+3 \frac{I_{3}}{I_{1}} A^{2}\right)\right]^{2}}{\left(1+\frac{9}{4} \frac{I_{2}}{I_{0}} A^{2}\right)}\right)^{1 / 2} .
$$

One may use this relation to compute $v_{0}$ for a range of values of $A$ and $\omega$ of interest - typically $A \in] 0,1\left[\right.$ and $\omega \in[1-\sigma, 1+\sigma]$. The contour lines of $v_{0}$ may then be plotted using numerical approximation methods (e.g. the contour function in Matlab), yielding the frequency response of the system for various values of $v_{0}$.

\section{MBP method}

Equation (4) is multiplied by $\left(1-a w_{C C}\right)^{2}$, yielding:

$$
\left(a+\frac{\dot{a}}{Q}+\ddot{a}\right) w_{C C}\left(1-a w_{C C}\right)^{2}=\eta\left(1+2 v_{0} \cos (\omega \tau)\right)
$$

and projected on $w_{C C}$. This results in the lumped model:

$$
\left(a+\frac{\dot{a}}{Q}+\ddot{a}\right)\left(I_{1}-2 a I_{2}+a^{2} I_{3}\right)=\eta\left(1+2 v_{0} \cos (\omega \tau)\right) I_{0} .
$$

The method of averaging results in:

$$
\left\{\begin{array}{c}
A\left(1-\omega^{2}\right)\left(1+\frac{3}{4} \frac{I_{3}}{I_{1}} A^{2}\right)=-2 \eta v_{0} \sin \varphi \frac{I_{0}}{I_{1}} \\
A \frac{\omega}{Q}\left(1+\frac{1}{4} \frac{I_{3}}{I_{1}} A^{2}\right)=2 \eta v_{0} \cos \varphi \frac{I_{0}}{I_{1}}
\end{array}\right.
$$

from which $v_{0}$ can be expressed as:

$$
v_{0}=\frac{I_{1}}{2 \eta I_{0}} A\left(\frac{\omega^{2}}{Q^{2}}\left(1+\frac{1}{4} \frac{I_{3}}{I_{1}} A^{2}\right)^{2}+\left(1-\omega^{2}\right)^{2}\left(1+\frac{3}{4} \frac{I_{3}}{I_{1}} A^{2}\right)^{2}\right)^{1 / 2}
$$




\section{API method}

Equation (4) is projected on $w_{C C}$. The right hand-side is approximated, following [10], as:

$\int_{0}^{1} \frac{w_{C C}}{\left(1-a w_{C C}\right)^{2}} d \xi \approx I_{0} \frac{1+\kappa a}{(1-a)^{3 / 2}}, \kappa \approx 2.15 \times 10^{-2}$,

which yields the following lumped model:

$\left(a+\frac{\dot{a}}{Q}+\ddot{a}\right) I_{1}=\eta\left(1+2 v_{0} \cos (\omega \tau)\right) I_{0} \frac{1+\kappa a}{(1-a)^{3 / 2}}$.

Using the method of averaging on (15) requires approximating further projection integrals (on $\sin (\omega \tau+\varphi)$ and $\cos (\omega \tau+\varphi))$, which can be expressed with complete elliptic functions, whose asymptotic behavior is well known. In particular, we find:

$$
\begin{aligned}
& \frac{\omega^{2 \pi / \omega}}{\pi} \int_{0}^{2} \sin (\omega \tau+\varphi) \frac{1+\kappa A \sin (\omega \tau+\varphi)}{(1-A \sin (\omega \tau+\varphi))^{3 / 2}} d \tau \approx \frac{A}{1-A^{2}}\left(\frac{3}{2}+\frac{3}{10} A^{2}\right) \\
& \frac{\omega^{2 \pi / \omega}}{\pi} \int_{0}^{2} \cos ^{2}(\omega \tau) \frac{1+\kappa A \sin (\omega \tau+\varphi)}{(1-A \sin (\omega \tau+\varphi))^{3 / 2}} d t \approx \frac{1}{A^{2}} \log \left(\frac{1}{1-A^{2}}\right)
\end{aligned}
$$

with a relative error between the left- and right-hand side of (16) (respectively (17)) smaller than $2 \%$ (respectively 6\%) for $A \in[0,0.99[$ and a bounded relative error as $A \rightarrow 1$. This yields the steady-state lumped model:

$$
\left\{\begin{array}{c}
A\left[\left(1-\omega^{2}\right)-\eta \frac{I_{0}}{I_{1}} \frac{15+3 A^{2}}{10\left(1-A^{2}\right)}\right]=-2 \eta v_{0} \sin \varphi \frac{I_{0}}{I_{1}} \frac{1}{A^{2}} \log \left(\frac{1}{1-A^{2}}\right) \\
A \frac{\omega}{Q}=2 \eta v_{0} \cos \varphi \frac{I_{0}}{I_{1}} \frac{1}{A^{2}} \log \left(\frac{1}{1-A^{2}}\right)
\end{array}\right.
$$

and the following expression for $v_{0}(A, \omega)$ :

$$
v_{0}=\frac{I_{1}}{2 \eta I_{0}} A\left(\frac{\omega^{2}}{Q^{2}}+\left[\left(1-\omega^{2}\right)-\eta \frac{I_{0}}{I_{1}} \frac{15+3 A^{2}}{10\left(1-A^{2}\right)}\right]^{2}\right)^{1 / 2} \times \frac{A^{2}}{\log \left(\frac{1}{1-A^{2}}\right)} .
$$

Note that, because of the presence of the logarithm, (18) cannot be turned into a polynomial in $A^{2}$, as opposed to (7) and (11). However, the contour lines of $v_{0}(A, \omega)$ may easily be plotted.

\section{3 - Results}

The contour lines corresponding to the values of $v_{0}$ obtained with (8), (12) and (19) are plotted in Fig. 1. The parameter values are $\eta=0.01, Q=500$ and $v_{0} \in\{0.02,0.03,0.04\}$. 


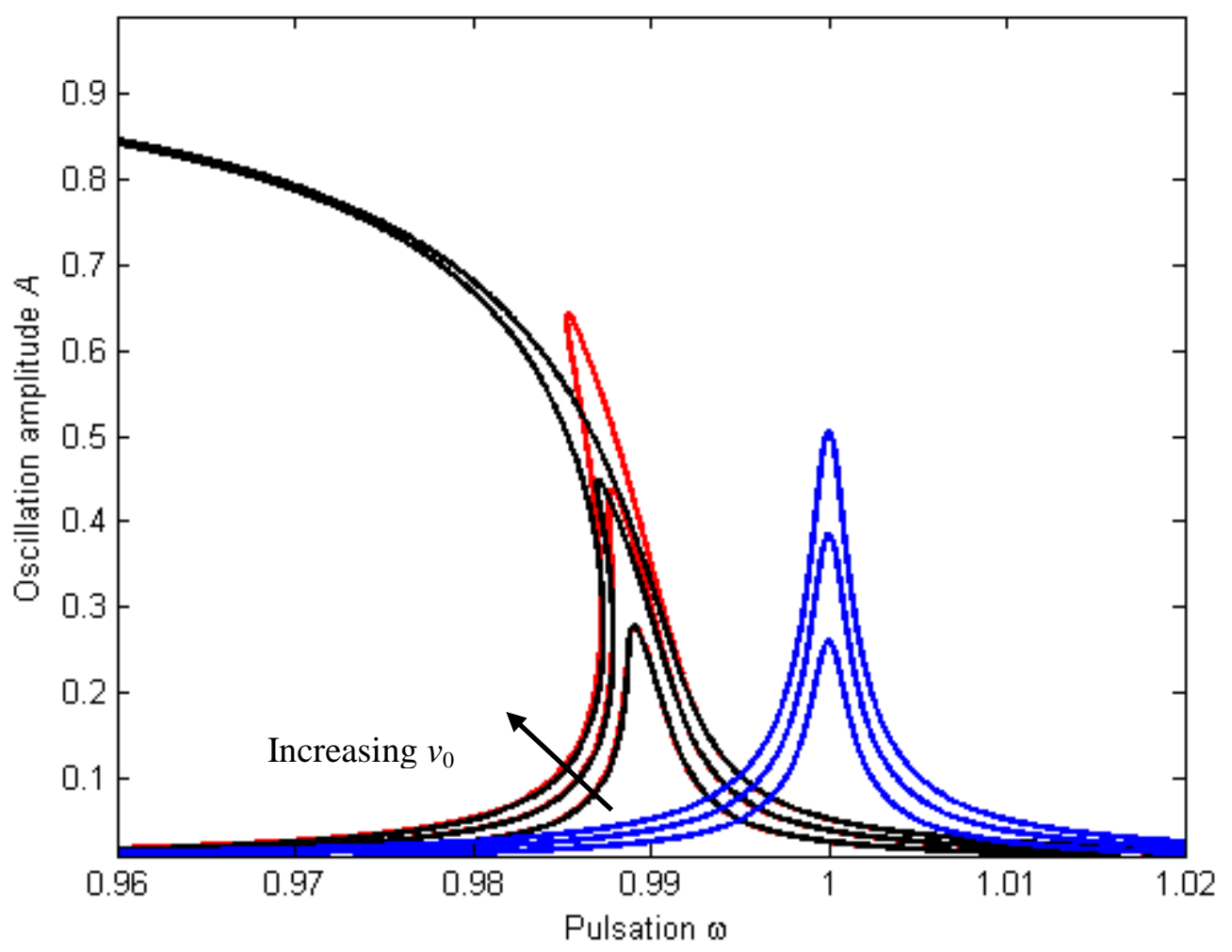

Fig. 1 - Steady-state amplitude for the clamped-clamped beam problem (1), as calculated by TS method ((8), red contour lines), MBP method ((12), blue contour lines) and API method ((19), black contour lines).

For the smallest excitation amplitude $\left(v_{0}=0.02\right)$, there is an excellent match between the API and TS methods, whereas they tend to disagree for large excitation amplitudes. The hysteretic character of the frequency response is more marked for the API method than for the TS method. The MBP method consistently disagrees with the other two methods. In particular, it is remarkable that the MBP method fails to capture:

- the down-shift of the resonance frequency caused by the DC bias, even at very small oscillation amplitudes.

- large amplitude effects such as the hysteretic characteristic of the frequency response.

Qualitatively similar results are obtained for other sets of parameters. In fact, it is clear from (11) that the MBP method predicts that resonance always occurs at $\omega=1$, regardless of $\eta, v_{0}$ or $A$.

Another interesting result is obtained when performing transient simulations of the nonlinear lumped parameter models (5), (10) and (15): provided one divides both sides of (10) by $I_{1}-2 a I_{2}+a^{2} I_{3}$, resulting in:

$a+\frac{\dot{a}}{Q}+\ddot{a}=\frac{\eta I_{0}}{I_{1}-2 a I_{2}+a^{2} I_{3}}\left(1+2 v_{0} \cos (\omega \tau)\right)$,

the three models have the structure of a linear $2^{\text {nd }}$-order system with a feedback nonlinearity and may readily be simulated with an ODE solver. The simulated frequency responses, obtained by slowly 


\section{SPECIAL SESSION ON NONLINEAR DYNAMICS OF MEMS AND NEMS or REGULAR SESSION ON REDUCED-ORDER MODELING - Oral presentation}

sweeping the excitation frequency up and down, are represented in Fig. 2. Quite surprisingly, there is a very strong qualitative difference between the results obtained with the MBP transient model (20) and the MBP steady-state model (12). On the other hand, the results obtained by simulating (5) and (15) are qualitatively and quantitatively coherent with those obtained with (8) and (19). Furthermore, there is an almost perfect match between:

- the frequency response obtained with the API method through transient simulation of (15).

- the frequency response obtained with the API method through steady-state analysis (19).

- the frequency response obtained by modal projection of (1) on the first 4 beam eigenmodes and transient simulation of the resulting system, the projection integrals being evaluated at each time step (Fig. 2).

This shows that the single-mode / single-harmonic approach is valid for capturing the nonlinear behavior of (1) close to resonance, even at very large oscillation amplitudes. These results are discussed in the next section.
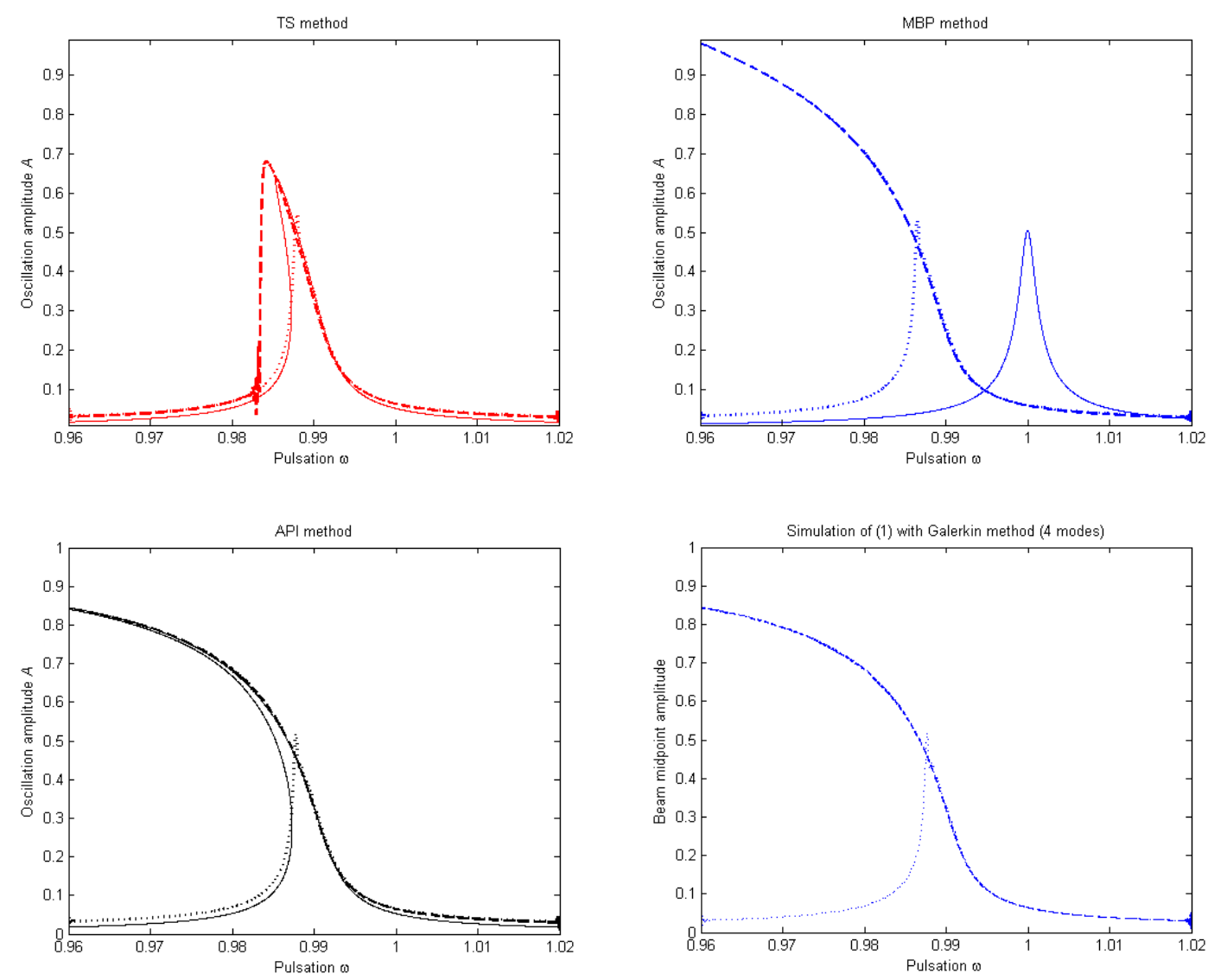

Fig. 2 - Frequency responses simulated with the transient lumped models (5), (20) and (15), for $\eta=0.01, Q=500$ and $v_{0}=0.04$. Dashed lines correspond to a down-sweep of the excitation frequency, dotted lines to an up-sweep. The plain line curves are taken from Fig. 1, for comparison. The bottom right picture shows results obtained by simulating (1) with a 4-eigenmode Galerkin method and evaluating the projection integrals at each time-step using a numerical quadrature scheme. 


\section{4 - Discussion}

To understand the failure of the MBP method in the steady-state sinusoidal regime, one should first make clear that using the method of averaging on (10) is the same as using the MBP method on (20), in the time domain instead of the space domain, since:

- (10) is obtained by multiplying (20) with the denominator of the right-hand side (the multiply step of the MBP method)

- the averaged steady-state equations are obtained by projection of (10) on $\sin (\omega \tau+\varphi)$ and $\cos (\omega \tau+\varphi)$.

As was already pointed out, the MBP method can accurately predict the static behavior of a clampedclamped beam only if the number of modes used for the decomposition (2) is large enough (on the order of 5, in [4]). It should then come as no surprise that the simulated steady-state behavior of (20) may be accurately predicted by MBP/averaging only if a large number of harmonics are used. Thus, the results of the MBP method in the steady-state sinusoidal regime should only be trusted when a large number of modes and a large number of harmonics are used. Unfortunately, in this case, the MBP method loses most of its interest because of the complexity of the calculations it involves.

On the other hand, the API method gives good qualitative and quantitative results. Establishing the model with the API method is a bit more costly than with the TS method, and it may be impossible to express the steady-state solutions as the roots of a polynomial. However, we have explained how the steady-state frequency responses may be plotted as the contour lines of $v_{0}$, with a very low computational burden.

Finally, the (often criticized) TS method gives good qualitative results, although it fails quantitatively. It may then be used safely for the qualitative analysis of resonant MEMS devices.

\section{References}

[1] J. F. Rhoads et al., "Generalized parametric resonance in electrostatically actuated microelectromechanical oscillators”, Journal of Sound and Vibration, vol. 296, pp. 797-829, 2006

[2] J. Chen et al., "Reduced-order modeling of weakly nonlinear MEMS devices with Taylor-series expansion and Arnoldi approach", Journal of Microelectromechanical Systems, vol. 13, pp. 441-451, 2004

[3] A. A. Trusov et al., "Capacitive detection in resonant MEMS with arbitrary amplitude of motion", Journal of Micromechanics and Microengineering, vol. 17, pp. 1583-1592, 2007

[4] M. I. Younis et al., "A reduced-order model for electrically actuated microbeam-based MEMS", Journal of Microelectromechanical Systems, vol. 12, pp. 672-680, 2003

[5] A. H. Nayfeh et al., "Reduced-order models for MEMS applications", Nonlinear Dynamics, vol. 41, pp. 211-236, 2005

[6] M. J. Snow et al., "Comprehensive reduced-order models of electrostatically actuated MEMS switches and their dynamics including impact and bounce", ASME IDETC/CIE 2010, 10 pages. 
SPECIAL SESSION ON NONLINEAR DYNAMICS OF MEMS AND NEMS or REGULAR

SESSION ON REDUCED-ORDER MODELING - Oral presentation

[7] N. Kacem et al., "Computational and quasi-analytical models for non-linear vibrations of resonant MEMS and NEMS sensors", International Journal of Non-Linear Mechanics, vol. 46, pp. 532-542, 2011

[8] S. Chatterjee et al. "A large deflection model for the pull-in analysis of electrostatically actuated microcantilever beams", Journal of Sound and Vibration, vol. 322, pp. 969-986, 2009

[9] L. D. Gabbay et al., "Computer-aided generation of nonlinear reduced-order dynamic macromodels", Journal of Microelectromechanical Systems, vol. 9, pp. 262-269, 2000

[10] J. Juillard et al., "Modeling of micromachined beams subject to nonlinear restoring or damping forces", Journal of Microelectromechanical Systems, vol. 20, pp. 165-177, 2011

[11] A. Gelb, W. Van der Velde, "Multiple-input describing functions and nonlinear system design", McGraw-Hill, New-York, 1968 